\title{
Nonlinear asteroseismology: insight from amplitude and frequency modula- tions of oscillation modes in compact pulsators from Kepler photometry
}

\author{
Weikai Zong ${ }^{1,2, \star}$, Stéphane Charpinet ${ }^{1,2, \star}$, Gérard Vauclair ${ }^{1,2, \star}$, Noemi Giammichele $^{1,2,3}$, and Valérie Van Grootel ${ }^{4}$ \\ ${ }^{1}$ CNRS, IRAP, 14 avenue Edouard Belin, F-31400 Toulouse, France \\ ${ }^{2}$ Université de Toulouse, UPS-OMP, IRAP, Toulouse F-31400, France \\ ${ }^{3}$ Département de Physique, Université de Montréal, CP 6128, Succursale Centre-Ville, Montréal, QC H3C 3J7, Canada \\ ${ }^{4}$ Institut d'Astrophysique et de Géophysique, Quartier Agora, Allée du 6 Août 19c, 4000 Liège, Belgium.
}

\begin{abstract}
Nonlinear mode interactions are difficult to observe from ground-based telescopes as the typical periods of the modulations induced by those nonlinear phenomena are on timescales of weeks, months, even years. The launch of space telescopes, e.g., Kepler, has tremendously changed the situation and shredded new light on this research field. We present results from Kepler photometry showing evidence that nonlinear interactions between modes occur in the two compact pulsators KIC 8626021, a DB white dwarf, and KIC 10139564, a short period hot B subdwarf. KIC 8626021 and KIC 10139564 had been monitored by Kepler in short-cadence for nearly two years and more than three years without interruption, respectively. By analyzing these high-quality photometric data, we found that the modes within the triplets induced by rotation clearly reveal different behaviors: their frequencies and amplitudes may exhibit either periodic or irregular modulations, or remain constant. These various behaviors of the amplitude and of the frequency modulations of the oscillation modes observed in these two stars are in good agreement with those predicted within the amplitude equation formalism in the case of the nonlinear resonant mode coupling mechanism.
\end{abstract}

\section{Introduction}

Evolved compact pulsators, white dwarf and hot B subdwarf ( $\mathrm{sdB})$ stars, have been well investigated by the technique of asteroseismology. White dwarf stars are the end fates of stellar evolution for $98 \%$ of the stars in our Galaxy. They span over quite a large region in the H-R diagram, with effective temperature of $\sim 10000-170000 \mathrm{~K}$ and surface gravity of $\sim 5.5-8.5$ dex $[1,2]$. SdB stars belong to the extreme horizontal branch in the H-R diagram, with a mass typically of $\sim 0.47 M_{\odot}$, effective temperature of $22000-40000 \mathrm{~K}$ and surface gravity of $\sim 5.2-6.1$ [3]. Observations from ground-based telescopes suggest that pulsation modes in compact pulsating stars may exhibit temporal variations in their amplitudes and/or frequencies, e.g., the GW Virginis variable star PG $0122+200$ [4]. However, it is difficult to observe one entire modulation cycle from ground as the modulations are typically much slower than the pulsation periods themselves [5].

The launch of space telescopes, such as Kepler, has tremendously changed the situations of uncovering the modulations of amplitude and frequency in pulsating stars. There are 113 candidates of compact stars that had been monitored by Kepler during its survey phase, aiming at searching for compact pulsators [6]. In the original Kepler mission, the final number of evolved compact pulsators reaches 24 , including 6 pulsating white dwarf star and 18

\footnotetext{
^e-mail: [weikai.zong,stephane.charpinet,gvauclair]@irap.omp.eu
}

pulsating sdB stars, see., e.g., Ref. [7]. Most of these 24 stars, in particular for sdB pulsators, have been observed by Kepler for more than two years without interruption. They are suitable candidates to observe the variability and stability in their oscillation modes.

In this proceeding, we assemble the results of first evidences of amplitude and frequency modulations of multiplet modes in two of these 24 compact stars, a Heliumatmosphere white dwarf, KIC 8626021, and a short-period V 361 Hya stars, KIC 10139654, pointing toward nonlinear mode interactions. These findings suggest that Kepler opens a new era to investigate nonlinear asteroseismology.

\section{Nonlinear resonant mode couplings}

The theoretical explorations of nonlinear asteroseismology, described in the framework of the amplitude equation (AE) formalism, began around 1980's. It concerned the mode resonance in the adiabatic and nonadiabatic cases $[8,9]$. A specific case of nonlinear AEs had been then applied to nonradial triplet modes that are induced by stellar rotation [10].

Within nonlinear asteroseismology, the stellar oscillations can be represented by temporal amplitudes as

$$
\mathbf{z}=\sum_{j} \frac{1}{2} A_{j}(t) e^{i \omega_{j} t} \mathbf{e}_{j}+C+O
$$


where $\mathbf{e}_{j}, \omega_{j}$, and $A_{j}(t)$ denote the linear nonadiabatic eigenvectors, eigenfrequency and the temporal amplitude of mode $j$, respectively, $C$ denotes complex conjugation and $O$ represents 'higher order terms'.

For an $\ell=1$ triplet, the nonlinear complex nonadiabatic AEs of the three modes are rewritten as a set [10],

$$
\begin{aligned}
\frac{d A_{-}}{d t}= & \kappa_{-} A_{-}+R_{-} A_{0}^{2} A_{+} \cos \left(\Phi-\delta_{-}\right) \\
& -A_{-}\left(q_{11} A_{-}^{2}+q_{12} A_{0}^{2}+q_{13} A_{+}^{2}\right), \\
\frac{d A_{0}}{d t}= & \kappa_{0} A_{0}+R_{0} A_{0} A_{+} A_{-} \cos \left(\Phi+\delta_{0}\right) \\
& -A_{0}\left(q_{21} A_{-}^{2}+q_{22} A_{0}^{2}+q_{23} A_{+}^{2}\right), \\
\frac{d A_{+}}{d t} & =\kappa_{+} A_{+}+R_{+} A_{0}^{2} A_{-} \cos \left(\Phi-\delta_{+}\right) \\
& -A_{+}\left(q_{31} A_{-}^{2}+q_{32} A_{0}^{2}+q_{33} A_{+}^{2}\right), \\
\frac{d \Phi}{d t} & =\delta \omega-2 R_{0} A_{-} A_{+} \sin \left(\Phi+\delta_{0}\right) \\
& +A_{0}\left[R_{-} \frac{A_{+}}{A_{-}} \sin \left(\Phi-\delta_{-}\right)+R_{+} \frac{A_{-}}{A_{+}} \sin \left(\Phi-\delta_{+}\right)\right],
\end{aligned}
$$

where the combination phase $\Phi=\phi_{+}+\phi_{-}-2 \phi_{0}, \kappa_{i}$ denote the linear growth rates, $q_{i j}$ and $R_{i}$ are the coupling coefficients, the phase shifts $\delta_{i}$ with the relation of $r_{i}=R_{i} \exp \left(i \delta_{i}\right)$, and the frequency mismatch $\delta \omega=$ $\omega_{+}+\omega_{-}-2 \omega_{0}$.

The numeric explorations of the above AEs reveal three distinct regimes of amplitude and frequency modulations of the interacting modes [10]:

a) The nonlinear frequency lock regime. The nonlinear couplings force the frequencies to be well equally spaced in the triplet modes, $\delta \omega=0$. The amplitudes are all constant and typically asymmetric, $A_{-} \neq A_{+}$.

b) The intermediate regime. The resonance modes exhibit amplitude and frequency modulations that can be periodic, multi-periodic, irregular, or even chaotic, depending on the real parameters of the star. When the periodic modulations occur, the period of the modulations is about the inverse of the frequency mismatch, $1 / \delta \omega$, or $\sim 1 / \kappa$.

c) The nonresonant regime. The nonlinear coupling effects are very small and the linear frequencies are very close to the linear results.

In the case of the three-mode resonance condition where $\omega_{1} \sim \omega_{2}+\omega_{3}$, the amplitude equation within the adiabatic approximation can be written as,

$$
\begin{aligned}
& \frac{d A_{1}}{d t}=\kappa_{1} A_{1}+\frac{i q}{\omega_{1} I_{1}} A_{2} A_{3} e^{-i \delta \omega t}, \\
& \frac{d A_{2}}{d t}=\kappa_{2} A_{2}+\frac{i q}{\omega_{2} I_{2}} A_{1} A_{3}^{*} e^{-i \delta \omega t}, \\
& \frac{d A_{3}}{d t}=\kappa_{2} A_{3}+\frac{i q}{\omega_{3} I_{3}} A_{1} A_{2}^{*} e^{-i \delta \omega t} .
\end{aligned}
$$

where $A_{j}^{*}$ stands for the complex conjugate of $A_{j}$, the frequency mismatch is $\delta \omega=\omega_{1}-\omega_{2}-\omega_{3}$, term $I_{j}$ are the mode inertia, the nonlinear coupling coefficient $q$ is very complicated and can be found in [8].

The numerical results suggest that the involved modes can be stable or modulate depending on the type of resonance and parameters such as their linear growth (damping) rates and the frequency mismatch [8].

\section{Amplitude and frequency modulations}

We have observed clear amplitude and frequency variations in two compact pulsators with photometry from the original Kepler mission. As space is limited, we only show here the modulations in the primary triplets in the two compact stars, KIC 8626021, a DB pulsating white dwarf, and KIC 10139564, a short-period pulsating sdB star. One can find more details and interesting mode behaviors in the two recently published papers $[11,12]$.

KIC 8626021 had been continuously monitored by Kepler in short-cadence for nearly two years, from quarter Q10.1 to Q17.2. Figure 1 shows the observed amplitude and frequency variations in the primary triplet near $4310 \mu \mathrm{Hz}$ in that star. The results suggest that both amplitude and frequency have signatures of quasi-periodic patterns which is further confirmed by the best sinusoidal fitting by one pure sine wave. The derived periods are $580 \pm$ 23, $680 \pm 10$ and $610 \pm 43$ days for the retrograde mode, central mode and prograde mode, respectively. More interestingly, we also observed that the two side components seem to evolve nearly in phase with similar modulation period of $\sim 600 \mathrm{~d}$ and nearly in anti-phase with the central component (which exhibits a slightly longer modulation period of $\sim 700 \mathrm{~d}$ ) in this triplet.

KIC 10139564 had been contiguously observed by Kepler in short-cadence for more than three years, from quarter Q5.1 to Q17.2. Figure 2 shows the amplitude and frequency modulations of the primary $p$-mode triplet near $5760 \mu \mathrm{Hz}$ in that star. We note that the hyperfine structures of the triplet components are significantly broaden which possibly indicate that the modes are unstable. This is further illustrated by the results shown in the other panels. We observed that the components show clear amplitude and frequency modulations: quasi-periodic frequency variations in all components and quasi-periodic amplitude variations in two components. It is very suggestive that the frequencies of the two side components evolve in anti-phase, which becomes more evident after removing the long-term trend of the frequency variations through quadratic fittings [12].

\section{Discussions}

The observed periodic behaviors of the triplets discussed here are pointing toward the intermediate regime of nonlinear mode couplings where nonlinear interacting modes may have periodic amplitude and frequency modulations. We discuss the two cases below. More details for different mode behaviors observed in these two targets can be found in Ref. [11, 12], where cases of frequency locked, regular or irregular variations are observed. 


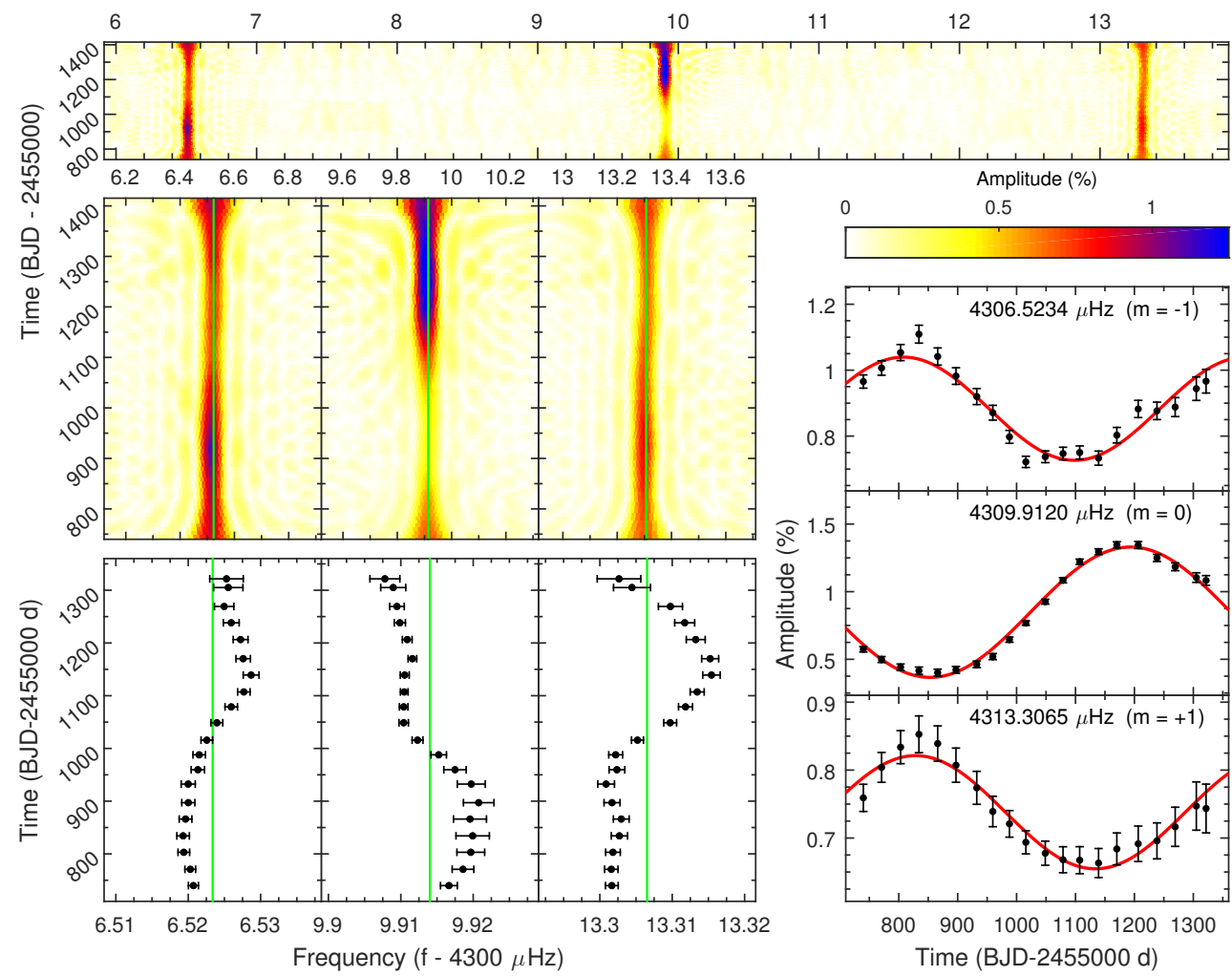

Figure 1: Amplitude and frequency modulations in the triplet near $4310 \mu \mathrm{Hz}$ in the DB white dwarf star KIC 8626021 . The color panels represent the sliding Lomb-Scargle periodogram. The vertical solid lines are the averaged values of the frequencies. Each data points in the bottom left and lower right panels are obtained from prewhitening subsets of the photometric data. The red solid curves denote the best sinusoidal fittings of amplitude variations. One can see more details in Ref. [11].
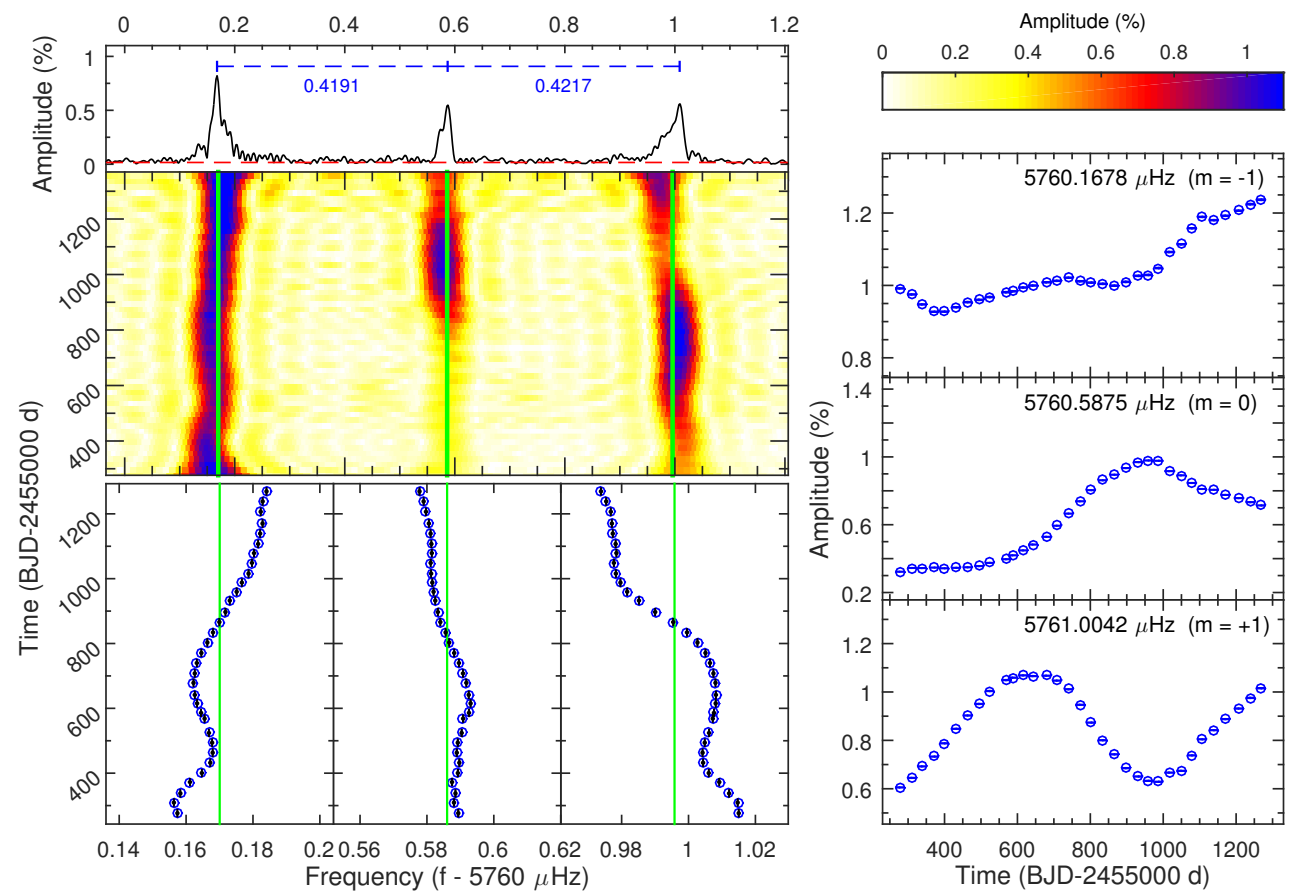

Figure 2: Similar as Fig. 1 but for the triplet modes in the short-period sdB star KIC 10139564 [12], except for the top panel. Top-left panel presents the hyperfine structure of the well defined triplet with near symmetric frequency spacing. We note that the error for each measurement is smaller than the symbol itself. 
For the triplet near $4310 \mu \mathrm{Hz}$ in KIC 8626021 , all the components show periodic amplitude and frequency variations on the timescale of $\sim 620 \mathrm{~d}$. This value is consistent with the theoretical expectation of $P_{m}($ th $) \sim 1 / \delta \omega_{t}=$ $620 \mathrm{~d}$. This supports the idea that we uncovered the right explanation for the mode behaviors in this triplet. However, as the coupling coefficients in the amplitude equations are unknown, we cannot conduct further quantitative comparisons between theoretical explorations and the observed modulations.

The triplet near $5760 \mu \mathrm{Hz}$ in KIC 10139564 shows quasi-periodic frequency and amplitude modulations that are also linked to the intermediate regime of nonlinear resonant mode couplings. The period of frequency variations in all the three components is about $580 \mathrm{~d}$ after removing the long-term trend [11]. This period should correspond to a value of $\sim 0.02 \mu \mathrm{Hz}$, the inverse of the frequency mismatch $\delta \omega$ in this triplet, which can be theoretically met when we consider the rotation up to second order for the $p$-modes [13]. The observed frequency asymmetry $\delta \omega_{o}$ is only $0.0026 \mu \mathrm{Hz}$ (see Fig. 2). However, we note that the maximum of the observed frequency mismatch of each subset data is about $0.02 \mu \mathrm{Hz}$. This result suggests that the nonlinear mode interactions possibly force the frequencies to vary around the near exact resonance.

\section{Conclusions}

The Kepler spacecraft provides unprecedented highquality and uninterrupted photometric data with a time baseline over a couple of years, which is very crucial to investigate behaviors of amplitude and frequency modulations of oscillation modes in pulsating stars. We study in depth the mode behaviors of triplet components in two compact pulsators from Kepler photometry, KIC 8626021, a DB white dwarf star, and KIC 10139564, a p-mode dominated hot B subdwarf star. We find clear periodic modulations of amplitude and frequency in two triplets in those two stars, that can be associated with the intermediate regime of nonlinear resonant coupling mechanism. The observed timescales of the modulations are consistent with the predictions of nonlinear amplitude equation formalism. We also observed a variety of mode behaviors in many multiplet modes in those two stars and more details can be seen in Ref. [11, 12]. These results are the first evidence of nonlinear mode interactions in pulsating compact stars.
We emphasize that the frequency variations induced by nonlinear effects could jeopardize any attempt to measure the secular rates of the pulsation period changes induced by evolutionary effect since the former one has a much larger influence on the pulsation periods than that from the latter one. To observe reliable change rates of the pulsation periods, one should be extremely careful in correctly extracting the frequencies and remove the nonlinear corrections beforehand.

These intriguing observed amplitude and frequency modulations, induced by nonlinear mode interactions, should bring significant constraints for further progress in the nonlinear stellar pulsation theory, for the development of new methods and techniques to prewhiten observed light curves, for the application of nonlinear resonance to other types of pulsating stars, and for the study of resonant mode behaviors observed from future space missions as well as ground-based telescopes, possibly, leading a new way to the "nonlinear asteroseismology".

\section{References}

[1] Fontaine, G., \& Brassard, P. 2008, PASP, 120, 1043

[2] Winget, D. E., \& Kepler, S. O. 2008, ARA\&A, 46, 157

[3] Heber, U. 2016, PASP, 128, 082001

[4] Vauclair, G., Fu, J.-N., Solheim, J.-E., et al. 2011, A\&A, 528, A5

[5] Goupil, M. J., Dziembowski, W. A., \& Fontaine, G. 1998, Baltic Astronomy, 7, 21

[6] Østensen, R. H., Silvotti, R., Charpinet, S., et al. 2010, MNRAS, 409, 1470

[7] Østensen, R. H., Reed, M. D., Baran, A. S., \& Telting, J. H. 2014, A\&A, 564, L14

[8] Dziembowski, W. 1982, Acta Astron., 32, 147

[9] Buchler, J. R., \& Goupil, M.-J. 1984, ApJ, 279, 394

[10] Buchler, J. R., Goupil, M.-J., \& Hansen, C. J. 1997, A\&A, 321, 159

[11] Zong, W., Charpinet, S., Vauclair, G., Giammichele, N., \& Van Grootel, V. 2016a, A\&A, 585, A22

[12] Zong, W., Charpinet, S., \& Vauclair, G. 2016b, A\&A, 594, A46

[13] Soufi, F., Goupil, M. J., \& Dziembowski, W. A. 1998, A\&A, 334, 911 